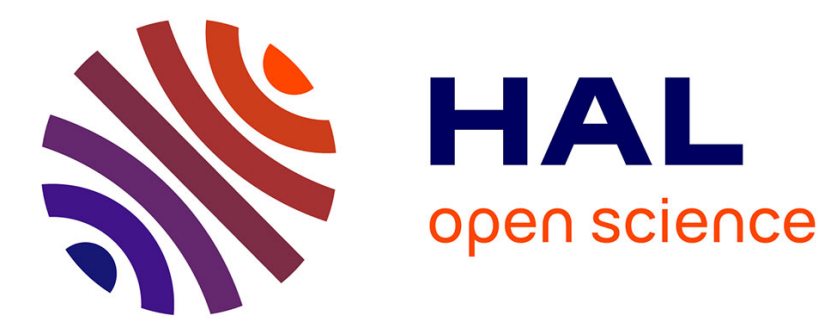

\title{
Large-Workspace Polyarticulated Micro-Structures Based-On Folded Silica for Tethered Nanorobotics
}

Yuning Lei, Cédric Clevy, Jean-Yves Rauch, Philippe Lutz

\section{To cite this version:}

Yuning Lei, Cédric Clevy, Jean-Yves Rauch, Philippe Lutz. Large-Workspace Polyarticulated MicroStructures Based-On Folded Silica for Tethered Nanorobotics. IEEE Robotics and Automation Letters, 2021, 7 (1), pp.88 - 95. 10.1109/LRA.2021.3118470 . hal-03414238v2

\section{HAL Id: hal-03414238 \\ https://hal.science/hal-03414238v2}

Submitted on 22 Nov 2021

HAL is a multi-disciplinary open access archive for the deposit and dissemination of scientific research documents, whether they are published or not. The documents may come from teaching and research institutions in France or abroad, or from public or private research centers.
L'archive ouverte pluridisciplinaire HAL, est destinée au dépôt et à la diffusion de documents scientifiques de niveau recherche, publiés ou non, émanant des établissements d'enseignement et de recherche français ou étrangers, des laboratoires publics ou privés. 


\title{
Large-Workspace Polyarticulated Micro-Structures Based-On Folded Silica for Tethered Nanorobotics
}

\author{
Yuning Lei, Cédric Clévy, Jean-Yves Rauch, Philippe Lutz
}

\begin{abstract}
Origami structures have a wide range of applications in robotics and have been intensively investigated by researchers in recent years. However, enabling sub-millimeter structures is an open question especially because of the lack of small enough joints. In this paper, compliant joints made of Silica by Focused Ion Beam (FIB) folding are proposed to achieve continuous, highly repeatable large motions. A polyarticulated structure including 3 joints is especially studied following a series of robotic analyses and experimentations to quantify the performances. The size of the structure firstly appears disruptive because smaller than $50 \mu \mathrm{m}$ in typical overall length, i.e. less than the radius of an optical fiber. Secondly, the structure can achieve a planar workspace of $57 \mu \mathrm{m}$ squared, which is significantly large compared to the structure dimension. Thirdly, repetitive movements performed at randomly selected positions, demonstrate an excellent repeatability standard deviations of 227 $\mathrm{nm}$ and $216 \mathrm{~nm}$ in $\mathrm{x}$ and $\mathrm{y}$ directions, respectively. These results together state the interest of novel polyarticulated structures resulting from the FIB folding as a basis for the next tethered nanorobotics generation.
\end{abstract}

Index Terms-Micro/Nano Robots, Actuation and Joint Mechanisms, Motion Control.

\section{INTRODUCTION}

A $\mathrm{S}$ a considerable amount of natural or artificially manufactured tiny objects such as cells, natural fibers [1], carbon nanotubes [2], and optical fibers have been increasingly used in recent decades for scientific, industrial and medical applications [3]-[11], a strong need for characterization systems and methods to manipulate at very small dimensional scales has arisen. Some commercial systems, such as the Atomic Force Microscope (AFM) have allowed significant advances in this regard [12], [13]. However, there are still substantial limitations due to the lack of dexterity and versatility in manipulation. That lead researchers to investigate another approach based on micro- and nanorobotics, The rapid development of micro- and nanoscale robotic devices [14] in recent years has demonstrated the great potential of this approach.

Manuscript received: April, 20, 2021; Revised July, 27, 2021; Accepted September, 26, 2021.

This paper was recommended for publication by Editor X. Liu upon evaluation of the Associate Editor and Reviewers' comments.

This work was supported by ISITE-BFC (contract ANR-15-IDEX-03), Equipex ROBOTEX (ANR-10-EQPX-44-01), ANR (ANR-19-CE10-000401), by Région Bourgogne Franche-Comté and the EIPHI Graduate School (ANR-17-EURE-0002).

The authors are with FEMTO-ST Institute, Univ. Bourgogne FrancheComté, UMR CNRS 6174, Besançon, France. yuning.lei demto-st.fr, cedric.clevyefemto-st.fr,

jy.rauchefemto-st.fr, philippe.lutzdfemto-st.fr

Digital Object Identifier (DOI): see top of this page.
To achieve multi-degree-of-freedom motion at the corresponding scale, the dimensions of the robotic system need to be as close as possible to the scale of the manipulation target to obtain sufficient manipulation dexterity. For nanorobotic applications, the size of their structures of interest is typically smaller than $100 \mu \mathrm{m}$ [3], [15], [16]. Further research and development is needed to achieve more advanced micro structures with experimentally measured large workspace and high repeatability to verify their feasibility of application to nanorobotics. Since the physics and chemistry of this scale are not completely understood, such work is of considerable practical difficulty [14], [15]. In the past decades, various attempts have been made to achieve such ultra-small-scale dexterous motion, here we cite some state-of-the-art research in Fig 1 , among them, the miniaturization of origami structures is one of the aspects with great potential.

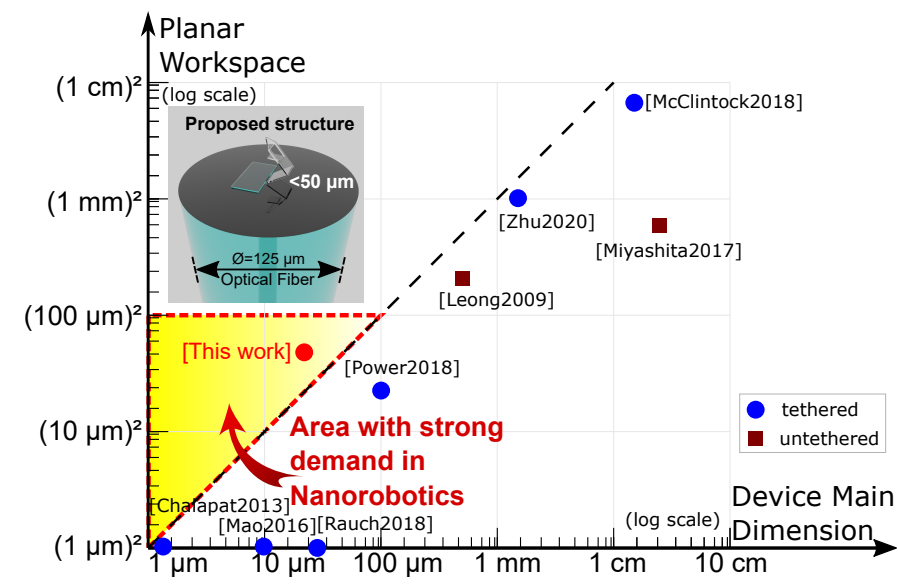

Fig. 1. Comparison of the device size and approximate planar workspace of some representative active origami robots and micro structures developed in recent years. The devices in the area above the dotted line indicate that they have a large workspace compared to their size. Among them, large workspace sub-100 $\mu \mathrm{m}$ devices are in high demand for the development of nanorobotics [3], [15], [16].

Origami-inspired folding techniques can convert a planar structure into a three-dimensional structure by folding, this feature makes origami well suited for improving the planarized structure design of conventional micro electro-mechanical systems (MEMS), the folds can be used as revolute articulations for robotic structure design. Some centimeter-scale origami structures have been proposed in recent years for robotic studies, for example, the milliDelta robot proposed by McClintock et al. [17] and the multilayer deformable structure fabricated by Miyashita et al. [18]. Their design of robots with folding as structural articulations practically demonstrated the feasibility 
and guaranteed motion performance of this folding technology applied to polyarticulated robot design. The emergence of millimeter-scale micro origami structures has generated a great deal of interest, Leong et al. [19] presented their untethered poly microgrippers with phalanges and joints, and Zhu et al. [20] proposed bilayer-hinge-based sub-millimeter micro-origami structures to demonstrate the elastic and plastic deformation of folding, allowing the structure to undergo complex deformations, showing the great potential for further miniaturization of articulated origami structures.

Rauch et al. [21] fabricated the world's smallest origami house with a overall dimension of about $20 \mu \mathrm{m}$ by applying a focused-ion-beam stress-induced deformation (FIB-SID) based self-folding process using a $1.2 \mu \mathrm{m}$ thick silica membrane and a $1 \mu \mathrm{m}$ thick lithium niobate membrane, respectively, on the cleaved surface of a single-mode fiber. The entire folding and assembly process was implemented in a SEM/FIB crossbeam vacuum station using a six-degree-of-freedom robotic manipulator. Their work has shown the feasibility of using rigid materials such as ceramics and silica, which are not traditionally collapsible, to create extremely small structures below $100 \mu \mathrm{m}$.

The work of Mao et al. [22], [23] demonstrates their method to control the angle of bi-directional folding of a $100 \mathrm{~nm}$ thick film between $-70^{\circ}$ and $90^{\circ}$ by controlling the irradiation of a focused ion beam, which shows that the FIB-
SID fabricated folding hinges have very small dimensions and a large angular range, and it is possible to obtain different hinges with similar thickness by controlling the folding to the same angle. From these works, we can see the prospect of designing and manufacturing more versatile polyarticulated robot structures.

However, the state-of-the-art research is still limited to the use of folds to form a specific angle in a passive origami structure, and there is no experimental evidence that such structures are capable of continuous motion within the angular range allowed by their mechanical strength, while the high repeatability required for nanomanipulations, typically less than $1 \mu \mathrm{m}[14]$, has not been verified in practice. To understand the suitability of such articulated structures for the development of nanorobotic applications, we still need to quantify the following aspect: Can such sub-100 $\mu \mathrm{m}$ articulated structures based on FIB-SID joints perform reversible, highly repeatable continuous motion over large workspaces?

To answer the question raised above, a sub-50 $\mu \mathrm{m}$ polyarticulated origami micro structure is introduced and studied in Section II, the structure is formed by cutting and self-folding a $900 \mathrm{~nm}$ thick fused silica membrane using a dual beam Scanning Electron Microscope (SEM)/Focused Ion Beam (FIB) station. The objective of this work is to investigate the angular range that each articulation of this FIB-SID polyarticulated origami structure can reach, so that the workspace of the

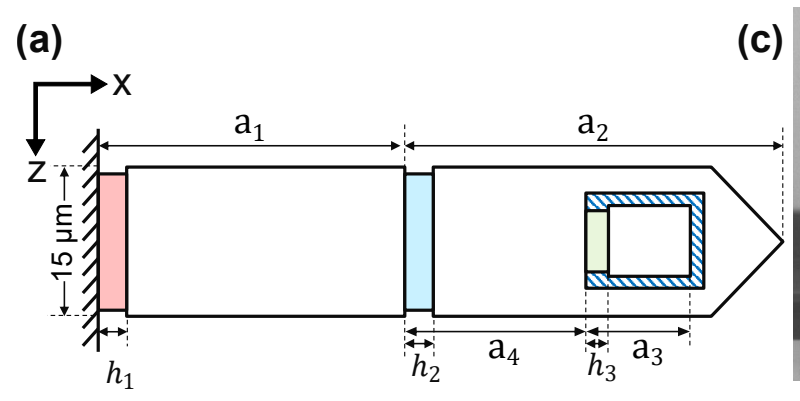

(b) (1)
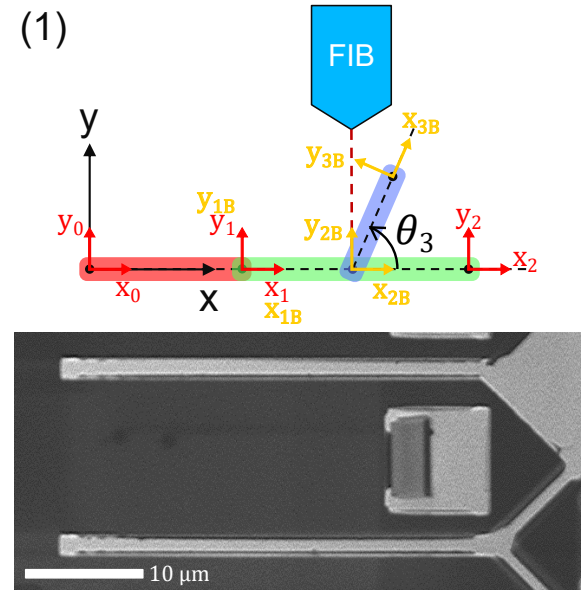

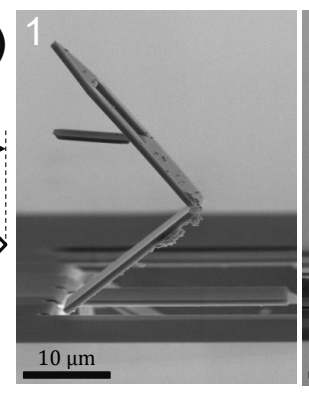

(2)
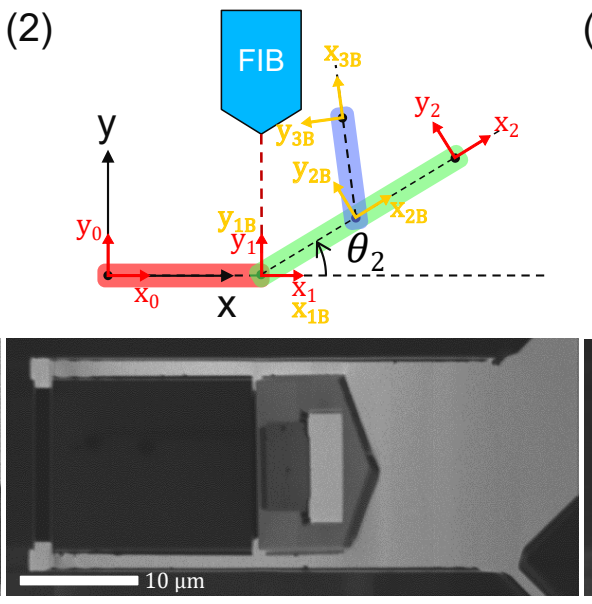
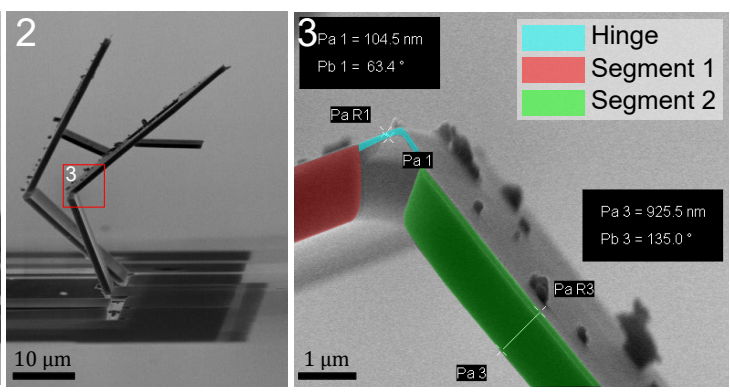

(3)
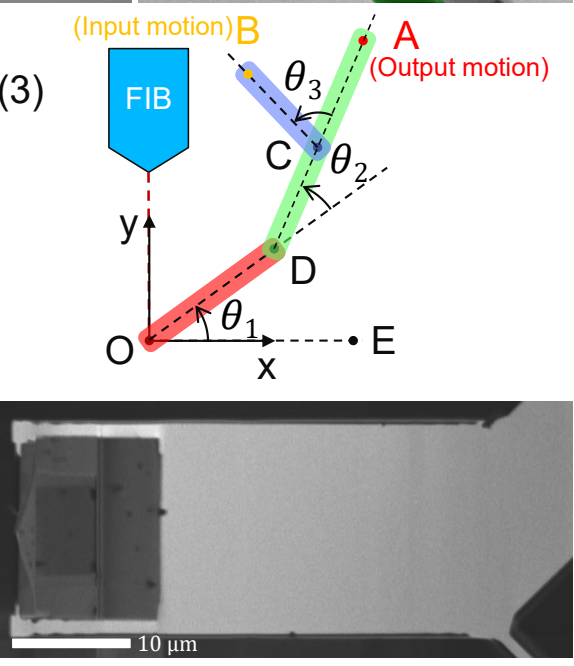

Fig. 2. (a) Planar Design of the micro-origami structure before folding. (b) The folding process of the origami: folding of each of the three rigid segments of the structure (1-3). schematic illustrations of side view, along with top view (FIB images), Scale bars are $10 \mu \mathrm{m}$. The two free ends of the second and third segment are called point $\mathrm{A}$ and point $\mathrm{B}$, respectively, and $\mathrm{C}, \mathrm{D}$ and $\mathrm{O}$ represent the articulations between segments. We define $\mathrm{E}$ as a certain point along the $\mathrm{x}$-axis for subsequent joint measurement and algebraic representation. The Denavit-Hartenberg (D-H) coordinate system of A and B are shown in red and yellow notations respectively in (1-2). (c) (1-2) Side view SEM images of the polyarticulated micro-origami structure after cutting and folding process completed. (3) Demonstrate the thickness of the silica membrane and of the folded hinges, measured as $925 \mathrm{~nm}$ and $104 \mathrm{~nm}$ respectively. 
structure's end-effector can be deduced through kinematic analysis in Section III and verified experimentally in terms of its workspace and repeatability, which are described in detail in Section $[\mathrm{IV}$ and $\mathrm{V}$ respectively.

\section{Foldable micro-ORigami Structures}

Quite a few studies related to origami design and manufacturing method in SEM/FIB station, especially the self-folding origami process by $\mathrm{Ga}^{+}$ion implantation, have been made in recent years, various structures of different shapes have been proposed, such as micro-cubic structures [24], flowershaped and spiral structures [25], [26]. In this work, a possible way of investigating the robotic properties of sub-50 $\mu \mathrm{m}$ polyarticulated origami structures is provided by using a sixdegree-of-freedom closed-loop robot manipulator under the vacuum chamber of the SEM, with a centimetric workspace and nanometric accuracy. Details of the manufacturing and manipulation of the polyarticulated micro-origami structure are presented separately in the following subsections.

\section{A. Manufacturing and folding of a polyarticulated micro- origami structure}

In this work, fused silica (silicon dioxide) has been used as a substrate membrane for cutting and folding the polyarticulated origami structure, and because it is a ceramic material and relatively easy to obtain homogeneous nanomembranes, its elastic behaviour is expected, which is important for the robotics, reversibility and repeatability of the robot's motions. Considering the technical difficulty of fabricating silica membranes below $1 \mu \mathrm{m}$ in thickness, we set the thickness of the silica membrane to $900 \mathrm{~nm}$ to ensure that the resulting membranes have homogenous thickness and to reduce the risk of fracture. The complete fused silica membrane is obtained by wet etching the oxidized silicon wafers with $\mathrm{HF}$ and $\mathrm{KOH}$ solutions.

As single folds have been demonstrated as feasible in previous research [21], [23], We desired a structure with at least three revolute articulations and which could work in the 2D plane to enable the characterisation of motion in SEM view. Fig. 2 demonstrates the design and fabrication process of the polyarticulated origami structure chosen as case study for the purpose of the objective of the study. A sub-50 $\mu \mathrm{m}$ long cantilever is designed to perform the FIB-induced folding and a triangular tip is created at the end-effector location for ease of subsequent measurements using computer vision methods. A small segment is designed to be cut and folded from the middle of the cantilever for welding with the stretched fiber tip. The thickness of the folded hinges has been measured to be approximately $100 \mathrm{~nm}$, hence, to ensure that they do not break during movement, the minimum segment width is set around $8 \mu \mathrm{m}$ to ensure the robustness of each hinge for repeatable folding, resulting in a width of $15 \mu \mathrm{m}$ for the entire cantilever. The length of the structure was limited to $50 \mu \mathrm{m}$ in order to comply with the target size requirements.
TABLE I

PARAMETERS OF THE ORIGAMI PROCESS FOR DIFFERENT SEGMENTS

\begin{tabular}{cccc}
\hline \hline Segment & Etching zone & Step 1: Trimming & Step 2: Folding \\
\hline \multirow{2}{*}{ a1 } & $\mathrm{x}=1.3 \mu \mathrm{m}$ & $\mathrm{T}=150 \mathrm{~s}$ & $\mathrm{~T}=30 \mathrm{~s}$ \\
& $\mathrm{Z}=16 \mu \mathrm{m}$ & $\mathrm{P}=30 \mathrm{kV}, 500 \mathrm{pA}$ & $\mathrm{P}=30 \mathrm{kV}, 200 \mathrm{pA}$ \\
a2 & $\mathrm{x}=1.3 \mu \mathrm{m}$ & $\mathrm{T}=150 \mathrm{~s}$ & $\mathrm{~T}=50 \mathrm{~s}$ \\
& $\mathrm{z}=16 \mu \mathrm{m}$ & $\mathrm{P}=30 \mathrm{kV}, 500 \mathrm{pA}$ & $\mathrm{P}=30 \mathrm{kV}, 100 \mathrm{pA}$ \\
a3 & $\mathrm{x}=1.3 \mu \mathrm{m}$ & $\mathrm{T}=100 \mathrm{~s}$ & $\mathrm{~T}=60 \mathrm{~s}$ \\
& $\mathrm{z}=9 \mu \mathrm{m}$ & $\mathrm{P}=30 \mathrm{kV}, 500 \mathrm{pA}$ & $\mathrm{P}=30 \mathrm{kV}, 50 \mathrm{pA}$ \\
\hline \hline
\end{tabular}

Consequently, a $45 \mu \mathrm{m} \times 15 \mu \mathrm{m}$ pattern with a triangular tip and a fixed end on the other side is cut on the fused silica membrane by the focused ion beam, shaping the body of the structure, a $6 \mu \mathrm{m} \times 8 \mu \mathrm{m}$ small segment $a_{3}$ was being cut and folded firstly from the part near the triangular tip. After this, the structure was divided into two segments of $a_{1}=20$ $\mu \mathrm{m}$ and $a_{2}=25 \mu \mathrm{m}$ in length and folded them in turn. The origami folding process of each segment was divided into two steps, with the first step using a stronger ion current to trim the silica membrane into thin hinges, followed by a weaker ion current to achieve a better control of the folding angle of each segment. The joint deformation limits of these hinges can be varied by changing their width $h_{1}, h_{2}$ and $h_{3}$, in our experiments, they were all set to $1.3 \mu \mathrm{m}$ to ensure a consistent angular range for each articulation. The FIB parameters of these origami folding process are described in Table $\mathbb{\Pi}$ for all three segments. The final angle of each articulation during the folding process needs to be set to a similar value of less than 90 degrees to ensure that the thickness of the flexible hinge is approximately the same and that they will not interfere with the subsequent folding of the other segments by obstructing the focused ion beam.

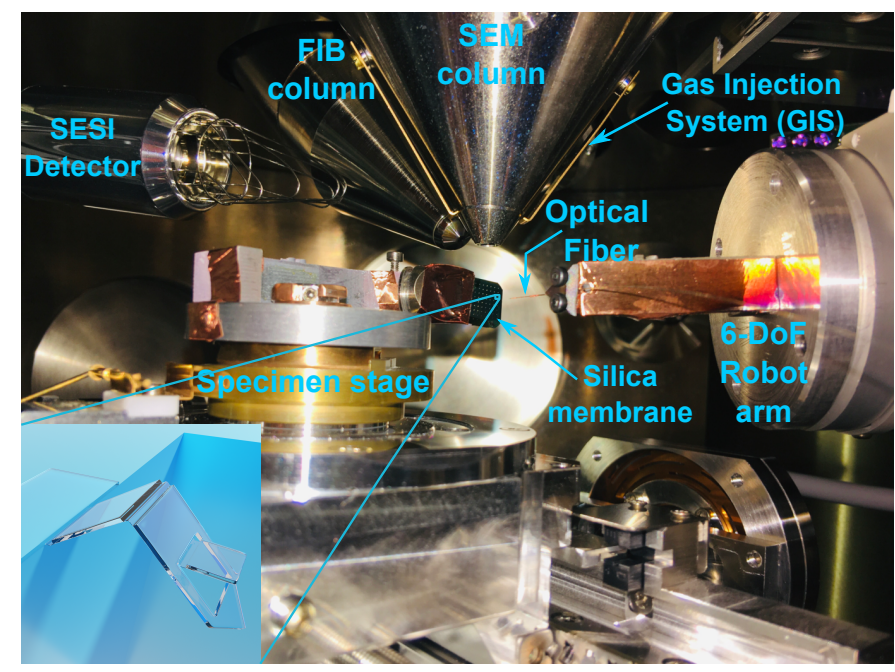

Fig. 3. Experimental configuration for welding and activating the polyarticulated origami structures with a stretched optical fiber tip. The locally zoomed-in rendering at the bottom left shows the micro origami structure folded on the silica membrane. 
B. Activating the polyarticulated micro-origami structure using a stretched optical fiber tip

The handling and deformation of the polyarticulated microorigami structure is controlled by a six-degree-of-freedom (6DoF) robotic manipulator, which is composed by SmarArt actuators in the vacuum chamber of the SEM. An optical fiber tip end with a stretched diameter of $1 \mu \mathrm{m}$ is mounted on the end-effector of the robot to activate the polyarticulated microorigami structure, as shown in Fig. 3 .

The two free ends of the second and third segment are called point $\mathrm{A}$ and point $\mathrm{B}$, respectively, corresponding to the output motion and the input motion end, shown in Fig. 2 b(3). In the experiment, the tip of the fiber was manipulated to contact point B on the third segment of the polyarticulated structure, and a Gas Injection System (GIS) was used to perform Ion Beam Induced carbon Deposition (IBID), carbon were deposited to weld the tip of the fiber to the third segment of the structure for reinforcement.

\section{MODELING}

In this section, the kinematic model of the proposed polyarticulated structure is presented in detail, demonstrating the relationship between the angle of each fold of the polyarticulated structure and the position of the end-effector obtained by using kinematic solutions. Each fold in the polyarticulated structure is regarded as a revolute joint for this purpose. This model is thereby used to simulate its workspace in the measured joint limits.

Following the definition of commonly used Denavit -Hartenberg (D-H) convention, the fixed link frame is defined as $\left\{x_{0}, y_{0}, z_{0}\right\}$, and the coordinate frame of Link $i$ is defined as $\left\{x_{i}, y_{i}, z_{i}\right\}$. The standard Denavit-Hartenberg (D-H) parameters of the polyarticulated origami structure considering the point $\mathrm{A}$ as end-effector are presented in Table $\mathrm{II}$ and the $\mathrm{D}-\mathrm{H}$ coordinate system is detailed in red notations in Fig 2 b)(1-2).

Considering the polyarticulated structure as a two-degreeof-freedom (2-DoF) planar serial chain with two revolute joints (RR), the kinematics equation ${ }^{0} T_{2}$ for the series chain of output motion point A can be obtained by using the lengths and angles of the three segments that make up the polyarticulated structure:

$$
{ }^{0} T_{2}={ }^{0} T_{1} \cdot{ }^{1} T_{2}
$$

Therefore, the transformation matrix of the end-effector A can be described as follows:

$$
\begin{aligned}
{ }^{0} T_{2} & =\left[\begin{array}{cccc}
c_{12} & -s_{12} & 0 & a_{2} c_{12}+a_{1} c_{1} \\
s_{12} & c_{12} & 0 & a_{2} s_{12}+a_{1} s_{1} \\
0 & 0 & 1 & 0 \\
0 & 0 & 0 & 1
\end{array}\right] \\
= & {\left[\begin{array}{cccc}
c_{\phi_{A}} & -s_{\phi_{A}} & 0 & x_{A} \\
s_{\phi_{A}} & c_{\phi_{A}} & 0 & y_{A} \\
0 & 0 & 1 & 0 \\
0 & 0 & 0 & 1
\end{array}\right] }
\end{aligned}
$$

Here we note $c_{i}=\cos \left(\theta_{i}\right), s_{i}=\sin \left(\theta_{i}\right), c_{i j}=$ $\cos \left(\theta_{i}+\theta_{j}\right), s_{i j}=\sin \left(\theta_{i}+\theta_{j}\right)$. So the output forward kinematic solution of the end-effector $\mathrm{A}$ can be obtained:

$$
\left\{\begin{array}{l}
x_{A}=a_{2} c_{12}+a_{1} c_{1} \\
y_{A}=a_{2} s_{12}+a_{1} s_{1} \\
\phi_{A}=\theta_{1}+\theta_{2}
\end{array}\right.
$$

TABLE II

STANDARD DENAVIT-HARTENBERG PARAMETERS OF ORIGAMI STRUCTURE WITH POINT A CONSIDERD AS END-EFFECTOR

\begin{tabular}{ccccc}
\hline \hline Link & $\begin{array}{c}\theta_{i} \\
\text { Joint angle }\end{array}$ & $\begin{array}{c}d_{i} \\
\text { Link offset }\end{array}$ & $\begin{array}{c}a_{i} \\
\text { Link length }\end{array}$ & $\begin{array}{c}\alpha_{i} \\
\text { Link twist }\end{array}$ \\
\hline 1 & $\theta_{1}$ & 0 & $a_{1}$ & 0 \\
2 & $\theta_{2}$ & 0 & $a_{2}$ & 0 \\
\hline \hline
\end{tabular}
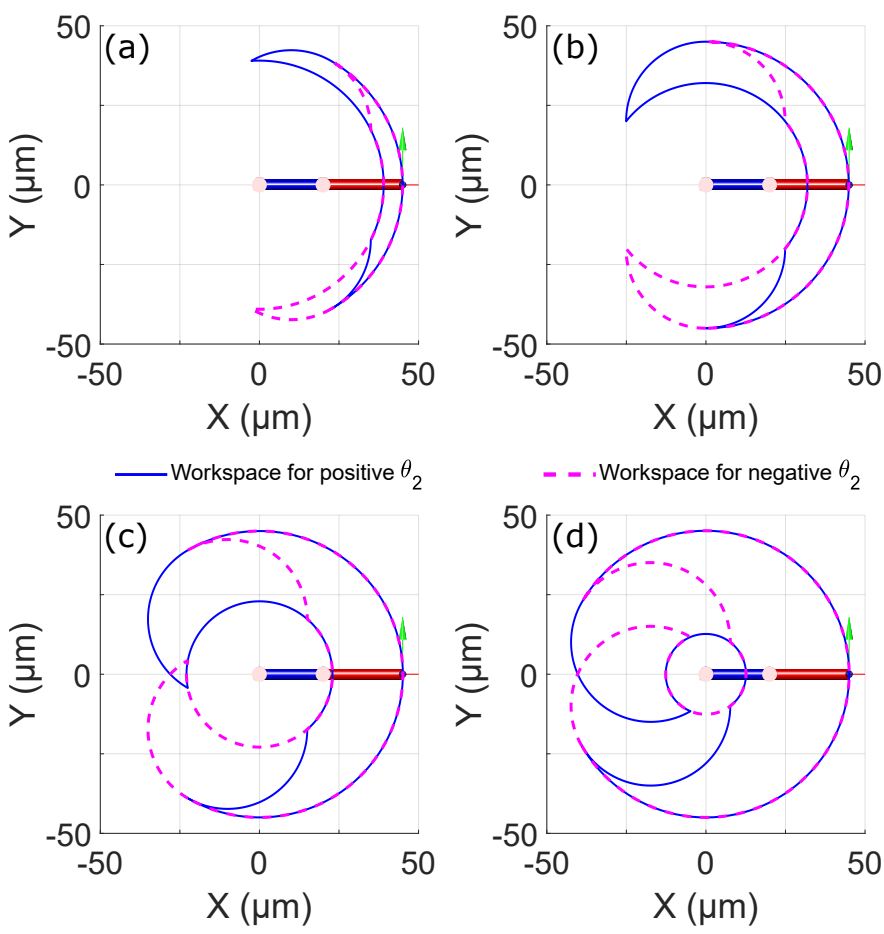

Fig. 4. Workspace simulation results obtained using analytical method, for different joint limits of $\theta_{1}$ and $\theta_{2}$. (a) $-60^{\circ} \leq \theta_{1}, \theta_{2} \leq 60^{\circ}$, (b) $-90^{\circ} \leq$ $\theta_{1}, \theta_{2} \leq 90^{\circ}$, (c) $-120^{\circ} \leq \theta_{1}, \theta_{2} \leq 120^{\circ}$, (d) $-150^{\circ} \leq \theta_{1}, \theta_{2} \leq 150^{\circ}$.

From these equations, the angular values can be used to get to the position of the point A by applying this forward kinematics solution. Based on the limitations of each flexible hinge, the workspace of the point A was also calculated, as shown in Fig. 4. The Robotic Toolbox [27] in Matlab was used to model a two-axis planar robot with the parameters in Table III. When using the analytical method [28], [29] to calculate the workspace, different resulting areas were obtained with positive or negative $\theta_{2}$ values respectively, with a partial area overlapped between them.

The angle of each revolute joint can be obtained by solving the inverse kinematic model (IKM) for the input motion of the point $\mathrm{B}$, and thus the position of the end-effector A can be obtained using the forward kinematic model (FKM). 


$$
\left(\begin{array}{c}
x_{B} \\
y_{B} \\
\phi_{B}
\end{array}\right) \stackrel{I K M}{\longrightarrow}\left(\begin{array}{c}
\theta_{1} \\
\theta_{2} \\
\theta_{3}
\end{array}\right) \stackrel{F K M}{\longrightarrow}\left(\begin{array}{c}
x_{A} \\
y_{A} \\
\phi_{A}
\end{array}\right)
$$

The D-H coordinate system parameters considering the point $\mathrm{B}$ as end-effector are presented in Table III and detailed in yellow notations in Fig $2(\mathrm{~b})(1-2)$. Especially, in order to distinguish with the coordinate system of point $A$, we define $\left\{x_{i B}, y_{i B}, z_{i B}\right\}$ as the Link $i$ frame of the $\mathrm{D}-\mathrm{H}$ coordinate system of point $\mathrm{B}$. Therefore the inverse kinematic solution can be obtained for point B using the iterative algorithm provided in the Robotic Toolbox.

According to the simulation results in Fig 4 , we can expect the structure proposed in section II to have a workspace of the same order of magnitude as the structure itself. This workspace depends very largely on the amplitude of each joint, the limits of the joints will be studied in the next section.

TABLE III

STANDARD DENAVIT-HARTENBERG PARAMETERS OF ORIGAMI STRUCTURE WITH POINT B CONSIDERD AS END-EFFECTOR

\begin{tabular}{ccccc}
\hline \hline Link & $\begin{array}{c}\theta_{i} \\
\text { Joint angle }\end{array}$ & $\begin{array}{c}d_{i} \\
\text { Link offset }\end{array}$ & $\begin{array}{c}a_{i} \\
\text { Link length }\end{array}$ & $\begin{array}{c}\alpha_{i} \\
\text { Link twist }\end{array}$ \\
\hline 1 & $\theta_{1}$ & 0 & $a_{1}$ & 0 \\
2 & $\theta_{2}$ & 0 & $a_{4}$ & 0 \\
3 & $\theta_{3}$ & 0 & $a_{3}$ & 0 \\
\hline \hline
\end{tabular}

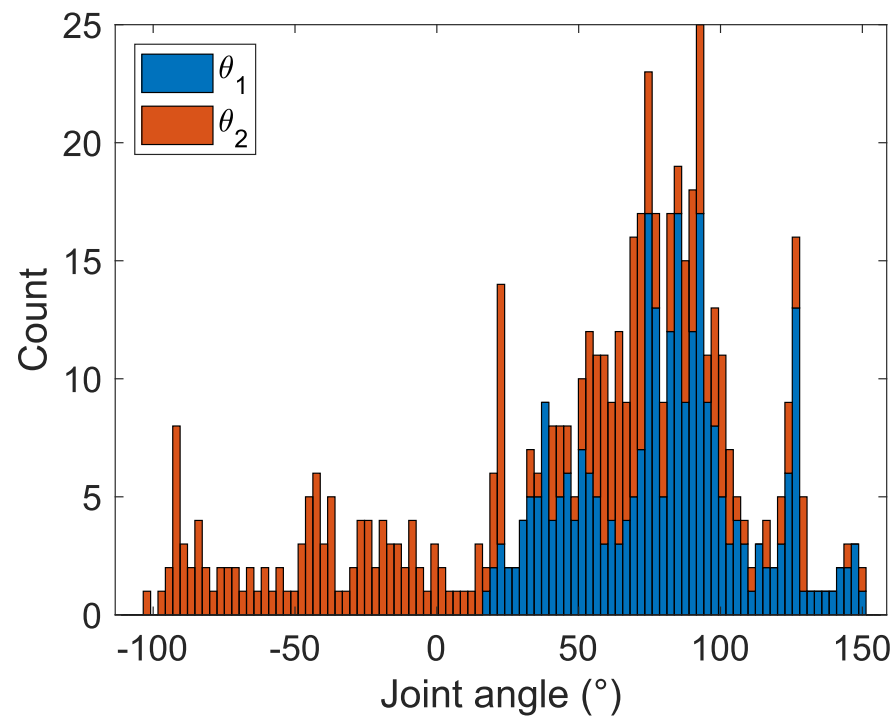

Fig. 5. Distribution of experimentally measured joint angle values of $\theta_{1}$ and $\theta_{2}$, The maximum joint angle obtained is $152.3^{\circ}$ and the minimum joint angle is $-101.6^{\circ}$

\section{EXPERIMENTAL WORKSPACE EVALUATION}

In this section, the attainable angle of articulation is quantified firstly in order to adapt the workspace of the polyarticulated origami structure obtained from the simulation to the actual joint limits, then the experimental procedure is described and the workspace is quantified experimentally to verify that the workspace obtained from the kinematic model is practically attainable.

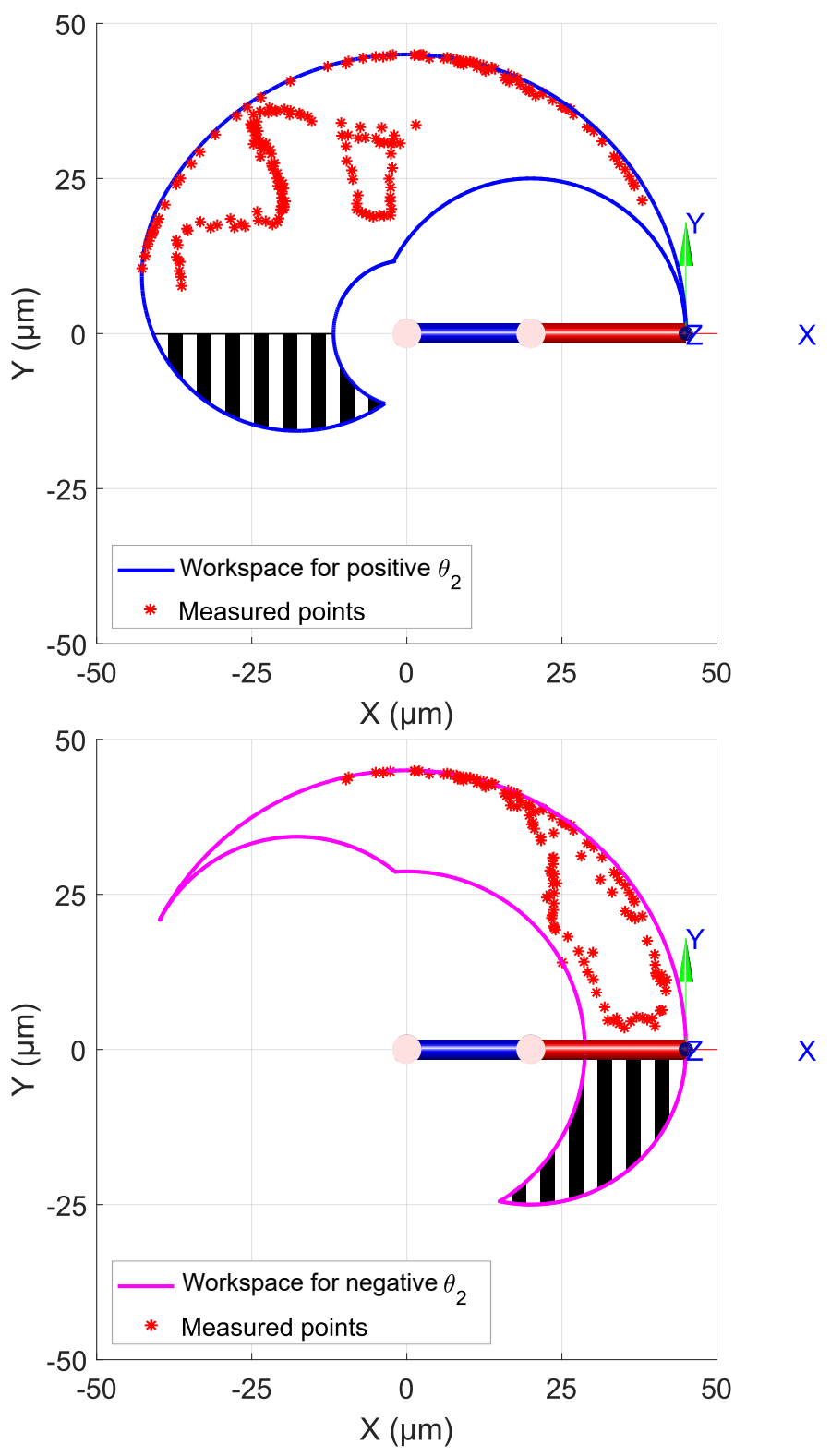

Fig. 6. Validation of the workspace of the polyarticulated origami structure: Simulation of the workspace of the validated joint confinement range (solid line) with the coordinates of the end-effector point A (red star marks) obtained from experimental measurements. The shaded part of the workspace cannot be verified due to the occlusion of the substrate

\section{A. Quantification of joint angle range}

By means of machine vision-based image correlation, the image pixel coordinates of the vertices of each rigid segment are measured and converted to coordinates in the work plane of the polyarticulated origami structure according to the pixel size. The placement of the sample has been adjusted so that the work plane of the structure is as perpendicular as possible to the direction of the scanning electron beam, making it easier to perform measurements and coordinate system conversions.

The positions of each rigid segment endpoint are measured and calculated using the cosine rule to obtain the angle values as shown in Equation 6, refer to Fig.2(b3), the sign of the result depends on whether the angle is folded forwards or backwards. 


$$
\begin{gathered}
\theta_{1}= \pm \cos ^{-1}\left(\frac{\overline{O D}^{2}+\overline{O E}^{2}-\overline{D E}^{2}}{2 \times \overline{O D} \times \overline{O E}}\right) \\
\theta_{2}= \pm\left[2 \pi-\cos ^{-1}\left(\frac{\overline{A D}^{2}+\overline{O D}^{2}-\overline{A O}^{2}}{2 \times \overline{A D} \times \overline{O D}}\right)\right] \\
\theta_{3}= \pm \cos ^{-1}\left(\frac{A C^{2}+\overline{B C}^{2}-\overline{A B}^{2}}{2 \times \overline{A C} \times \overline{B C}}\right)
\end{gathered}
$$

In the experiments, the maximum folding limit angle of each flexible hinge was measured to be $0 \leq \theta_{1} \leq 152.3^{\circ}$, $-101.6^{\circ} \leq \theta_{2} \leq 151.7^{\circ}$, distributions of the measurements of the joint angles are shown in Fig. 5 . Due to the obstruction of the field of view by the fused silica membrane, in practice, only the configuration of $\theta_{1}$ folding in the forward direction can be experimentally observed, so we use these measurement results as the joint range for this structure for the workspace analysis of the point A, hence the workspace calculated using these parameters is shown as solid envelope lines in Fig. 6

\section{B. Validation of the workspace}

The polyarticulated structure was activated to move with each joint varied in its measured joint range, a sequence of movements was obtained to validate the calculated workspace. Each motion step was performed with a movement of a few micrometers, and a set of images of the SEM view was saved for position measurement. Due to the limitations of the rotation angle, once the optical fiber tip is welded to the polyarticulated structure, the optical fiber tip was controlled to move only in the $\mathrm{x}$ and $\mathrm{y}$ directions without any rotation, therefore the entire workspace needs to be separated in two zones: the one with a positive $\theta_{2}$ and the other with a negative $\theta_{2}$. In these two cases, the welding direction of the fibers is different, so these two cases were experimented separately. The part of the workspace below the silica membrane could not be experimentally observed and verified due to the obstruction of the field of view by the membrane, Thus only the workspace above the membrane has been experimentally verified.

In Fig. 7 on the left side (a-d), several motion sequence
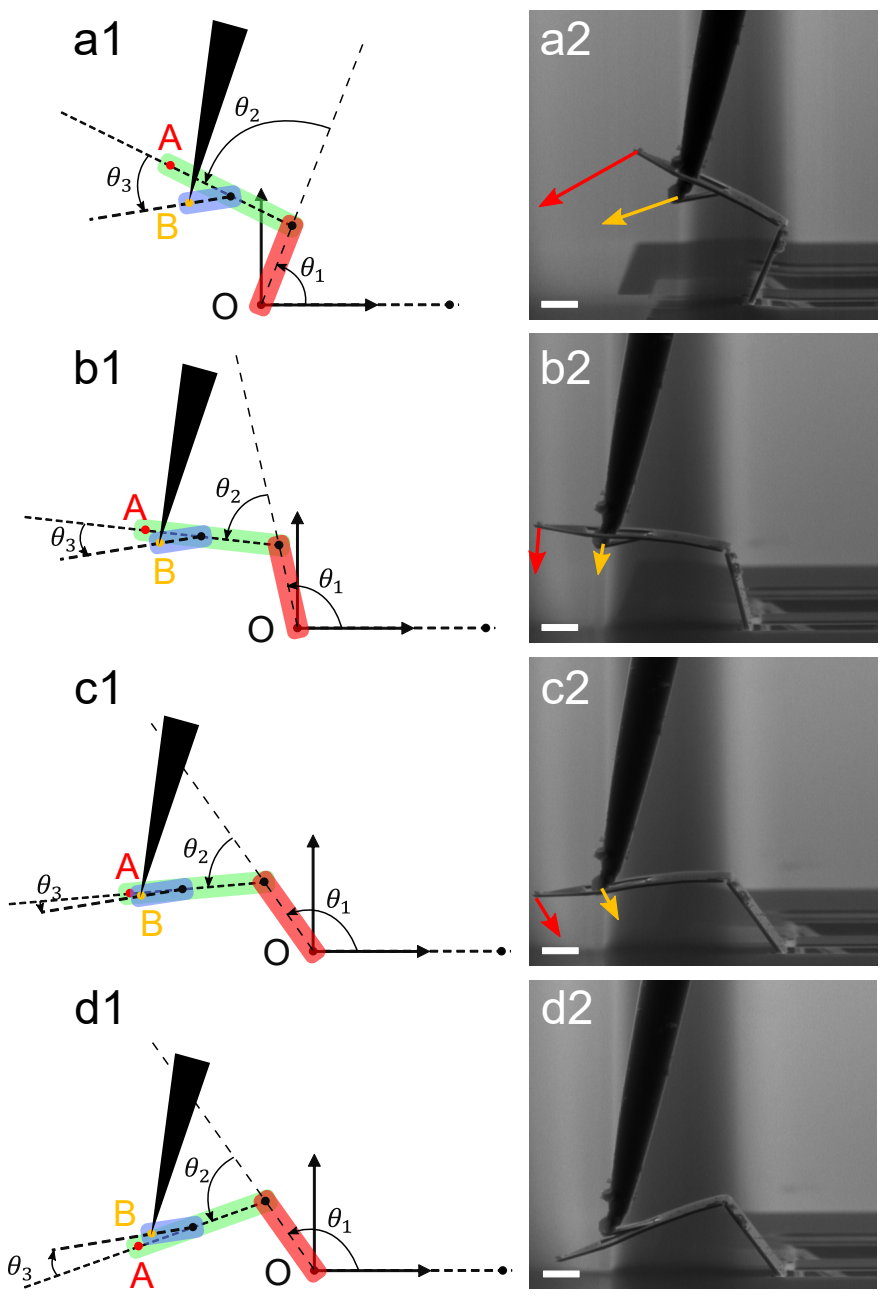
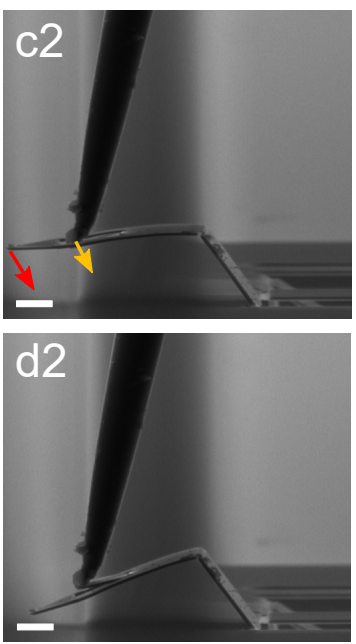
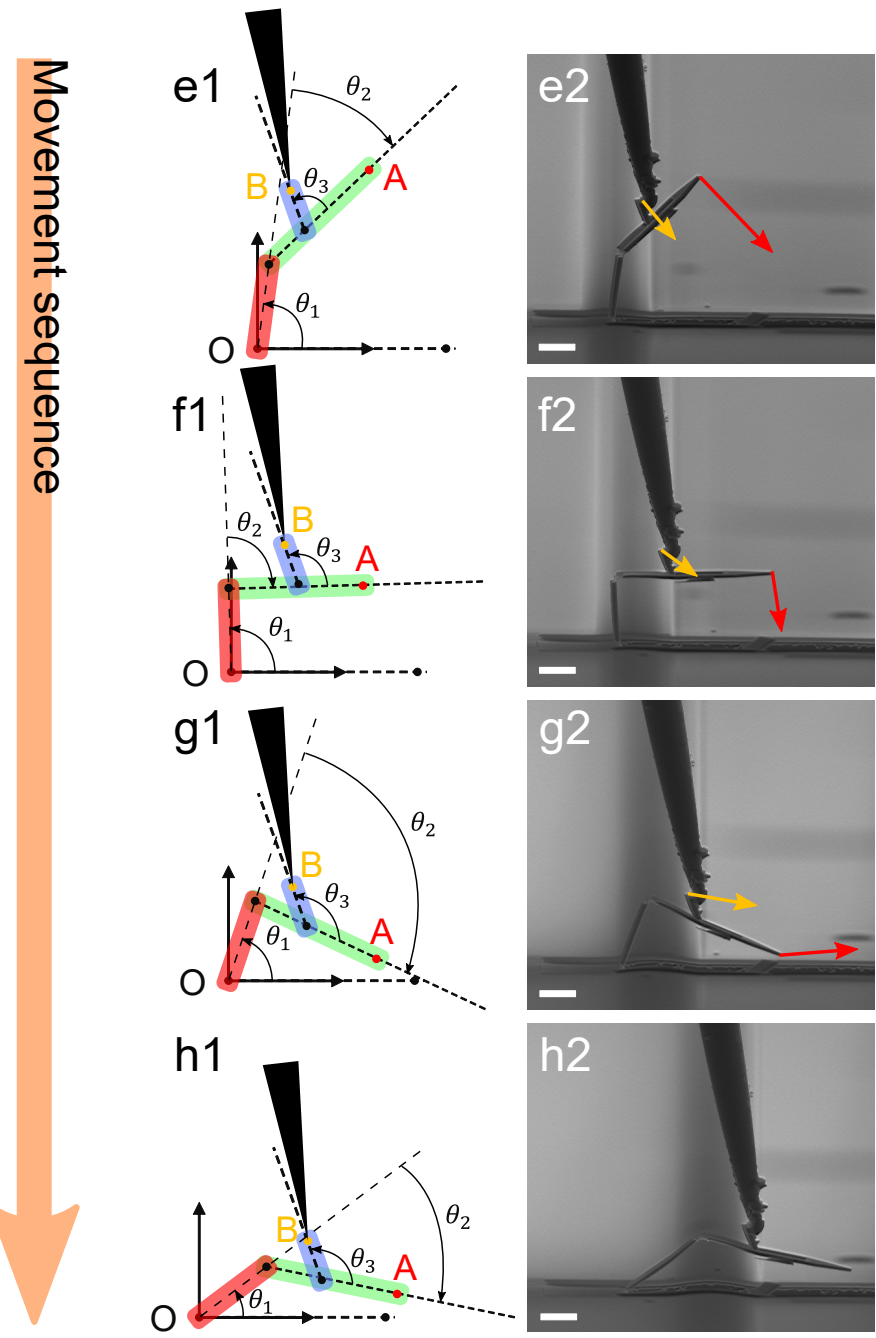

Fig. 7. Proof of concept of folding and actuation of the polyarticulated structure by the externally driven optical fiber. (a1)-(d1) Schematic representation of the changes of the joints during movement with $\theta_{2}$ folding in positive direction and (e1)-(h1) in negative direction. (a2)-(d2) Example of the SEM images obtained from the experiment for measuring and calculating the values of the angles and determining the position of the end-effector using visual methods, (e2)-(h2) are in negative direction. The arrows in the SEM images represent the direction of motion of point A (red) and point B (yellow) respectively. Scale bar is $5 \mu \mathrm{m}$ for all images. 
images are shown with $\theta_{2}$ folds in positive direction, and another case with $\theta_{2}$ folds in the opposite direction is represented on the right side of Fig. 7 (e-h). These images are used to characterize the joint angles and analysis the position of endeffector A.

The workspace of the end-effector A was verified by placing the measured points in the structure coordinate system as red marks in Fig. 6 The shaded area cannot be verified because it was shielded by the fused silica membrane and silicon substrate. The workspace achievable by the proposed polyarticulated micro-origami structure within the determined angular constraints was measured to be 57 micrometers squared, of which we verified a workspace of approximately 47.4 micrometers squared with experimental data.

\section{EXPERIMENTAL REPEATABILITY STUdY}

The repetitive movement experiences of determined poses performed by the proposed polyarticulated micro-origami structure are presented in this section to analyse the repeatability and reversibility of motions generation in nanoscale.

The joint parameters of the six-degree-of-freedom robot for a defined position in the workspace of the micro-origami structure were recorded, and twenty repetitive motions were made to this configuration, with sampling the end-effector position five times for each move to the saved position to reduce measurement uncertainty. Since the transformation between the discrete image coordinates system $R_{i}(u, v)$ in pixels and the robot workspace nanometric coordinates system $R_{r}(x, y)$ is calculated by Equation 7, The homogeneous coordinates of the end-effector $\mathrm{A}$ in the image coordinate system and the robot coordinate system are expressed respectively as $\left(u_{A}, v_{A}, 1\right)$ and $\left(x_{A}, y_{A}, 1\right)$, the image coordinates of the endeffector A were measured by the same vision-based method as being described in the previous section and obtain the point coordinates distribution within the robot's workspace by multiplying the coordinates by the size of each pixel $\left(k_{x}, k_{y}\right)$ in the image.

$$
\left[\begin{array}{c}
x_{A} \\
y_{A} \\
1
\end{array}\right]=\left[\begin{array}{ccc}
k_{x} & 0 & 0 \\
0 & k_{y} & 0 \\
0 & 0 & 0
\end{array}\right] \cdot\left[\begin{array}{c}
u_{A} \\
v_{A} \\
1
\end{array}\right] \cdot{ }^{i} T_{r}
$$

Here ${ }^{i} T_{r}$ is the transformation matrice from image coordinate system to the polyarticulated origami workspace coordinate system, $R_{r}(x, y)$ corresponding the one in Fig. 2 $\mathrm{b}, k_{x}$ and $k_{y}$ are the image nanometric pixel size in lateral and vertical directions.

The results of the measurements are shown in Fig. 8 , The positioning repeatability is defined by its standard deviation of all repeated positions to the mean position, in lateral, $s t d_{x}$, and in vertical, $s t d_{y}$.

$$
\bar{x}=\frac{1}{n} \sum_{j=1}^{n} x_{j}, \quad \bar{y}=\frac{1}{n} \sum_{j=1}^{n} y_{j}
$$

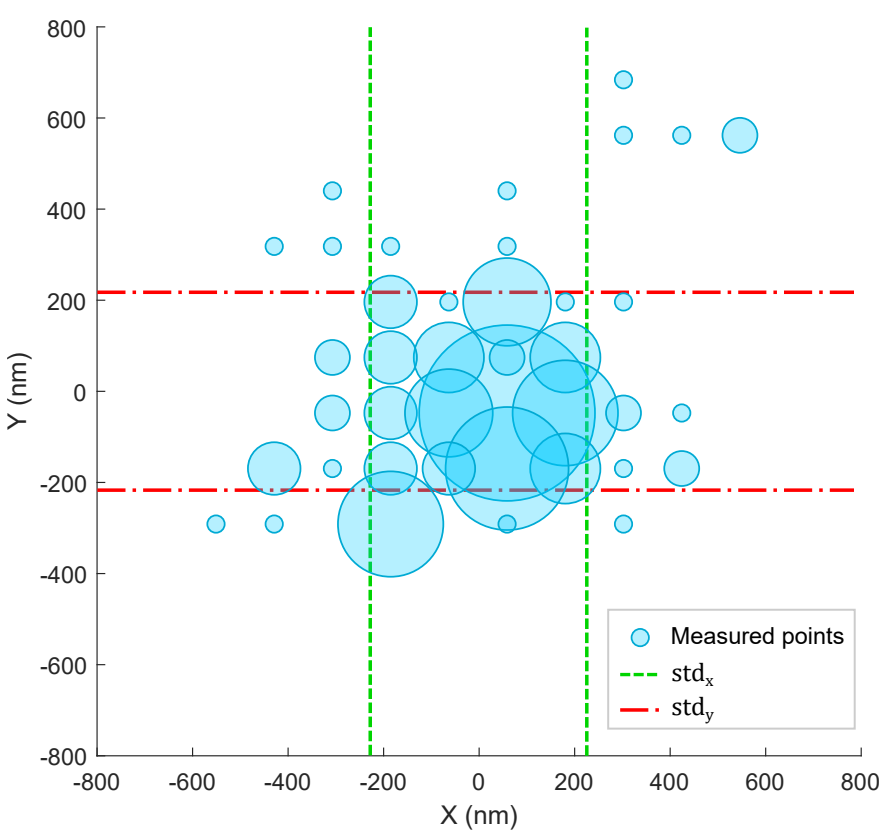

Fig. 8. Repeatability test, distribution of the results of 100 measurements for 20 repetitions of the determined pose (5 measurements per pose), The size of bubble indicates the density of the data measured at that point.

$$
\begin{aligned}
& s t d_{x}=\sqrt{\frac{\sum_{j=1}^{n}\left(x_{j}-\bar{x}\right)^{2}}{n-1}}, \\
& s t d_{y}=\sqrt{\frac{\sum_{j=1}^{n}\left(y_{j}-\bar{y}\right)^{2}}{n-1}}
\end{aligned}
$$

The experimental result shows that these repetitions follow a normal distribution of $227 \mathrm{~nm}$ and $216 \mathrm{~nm}$ standard deviation along $\mathrm{x}$ and $\mathrm{y}$ directions, respectively, for displacements of $20 \mu \mathrm{m}$. Due to limitations in image resolution and the effect of image distortion caused by the scanning electron beam, the five measurements of each single pose have an average error of $183 \mathrm{~nm}$, which is in the same order of magnitude as the experimental results. Accordingly, we can conclude that the intrinsic repeatability is limited by the conditions of measurement, the actual motion repeatability of the structure can be much better than the obtained experimental results.

\section{CONCLUSION}

In this article, we presented a sub-50 $\mu \mathrm{m}$ polyarticulated micro-origami structure made of a $900 \mathrm{~nm}$ thick fused silica membrane fabricated using FIB-induced folding process. The work demonstrated that this folded structure can induce reversible motions and thus the folded hinges can be considered as articulation. Every articulation can achieve a reversible large angle range between -101.6 and 152.3 degrees, and the polyarticulated structure is therefore able to reach a very large workspace of $57 \mu \mathrm{m}$ squared, and it has been verified that $47.4 \mu \mathrm{m}$ squared of this workspace is safe and experimentally achievable. The device size to workspace ratio was demonstrated at the same scale and makes it compatible with the considerable demand domains for large workspace nanorobotics 
devices smaller than 50 micrometers. Proposed polyarticulated structure enables reversible motions with a repeatability standard variance experimentally measured as $227 \mathrm{~nm}$ and $216 \mathrm{~nm}$ along the $\mathrm{x}$ and $\mathrm{y}$ directions, respectively, which demonstrates that this polyarticulated structure is suitable for motions and operations in nanorobotics, and thus provides the basis for subsequent design and fabrication of more dexterous sub-100 $\mu \mathrm{m}$ parallel robotic structures based on FIB-induced folding articulations, such as self-adaptive robotic fingers [30], for the next tethered nanorobotics generation.

\section{ACKNOWLEDGMENT}

This work was supported by the Equipex ROBOTEX (ANR-10-EQPX-44-01), the COLAMIR Project (contract ANR-19-CE10-0004-01), Région Bourgogne Franche-Comté, the EIPHI Graduate School (ANR-17-EURE-0002) and the French RENATECH network through its MIMENTO technological facility. The authors are also very grateful to Pierre Roux, Laurent Robert, Marion Vieira and Joël Agnus for their contribution in the design and fabrication of the wafers and experimental setup.

\section{REFERENCES}

[1] B. Han, V. Polonais, T. Sugi, R. Yakubu, P. M. Takvorian, A. Cali, K. Maier, M. Long, M. Levy, H. B. Tanowitz, G. Pan, F. Delbac, Z. Zhou, and L. M. Weiss, "The role of microsporidian polar tube protein 4 (PTP4) in host cell infection," PLOS Pathogens, vol. 13, no. 4, p. e1006341, apr 2017.

[2] C. Clevy, B. Sauvet, J.-Y. Rauch, O. Lehmann, F. Marionnet, P. Lutz, L. Beccacece, S. Xavier, R. Aubry, A. Ziaei, C. Prevot, and G. J. Laurent, "In-situ Versatile Characterization of Carbon NanoTubes using Nanorobotics," in 2019 International Conference on Manipulation, Automation and Robotics at Small Scales (MARSS). IEEE, jul 2019, pp. 1-6.

[3] B. Bhushan, Ed., Springer Handbook of Nanotechnology, ser. Springer Handbooks. Berlin, Heidelberg: Springer Berlin Heidelberg, 2010.

[4] M. Hwang and B. Yeom, "Fabrication of Chiral Materials in Nano- and Microscale," Chemistry of Materials, vol. 33, no. 3, pp. 807-817, feb 2021.

[5] P. Li, S. Chen, H. Dai, Z. Yang, Z. Chen, Y. Wang, Y. Chen, W. Peng, W. Shan, and H. Duan, "Recent advances in focused ion beam nanofabrication for nanostructures and devices: fundamentals and applications," Nanoscale, vol. 13, no. 3, pp. 1529-1565, 2021.

[6] J. Li, B. E.-F. De Ávila, W. Gao, L. Zhang, and J. Wang, "Micro/nanorobots for Biomedicine: Delivery, surgery, sensing, and detoxification," Science Robotics, vol. 2, no. 4, pp. 1-10, 2017.

[7] J. Zhang, O. Onaizah, K. Middleton, L. You, and E. Diller, "Reliable Grasping of Three-Dimensional Untethered Mobile Magnetic Microgripper for Autonomous Pick-and-Place," IEEE Robotics and Automation Letters, vol. 2, no. 2, pp. 835-840, 2017.

[8] L. Tan, A. C. Davis, and D. J. Cappelleri, "Smart Polymers for Microscale Machines," Advanced Functional Materials, vol. 31, no. 9, p. 2007125, feb 2021.

[9] M. Z. Miskin, A. J. Cortese, K. Dorsey, E. P. Esposito, M. F. Reynolds, Q. Liu, M. Cao, D. A. Muller, P. L. McEuen, and I. Cohen, "Electronically integrated, mass-manufactured, microscopic robots," Nature, vol. 584, no. 7822, pp. 557-561, 2020.

[10] B. Mauze, R. Dahmouche, G. J. Laurent, A. N. Andre, P. Rougeot, P. Sandoz, and C. Clevy, "Nanometer Precision with a Planar Parallel Continuum Robot," IEEE Robotics and Automation Letters, vol. 5, no. 3, pp. 3806-3813, 2020.

[11] X. Wang, C. Hu, S. Pane, and B. J. Nelson, "Dynamic Modeling of Magnetic Helical Microrobots," IEEE Robotics and Automation Letters, pp. 1-1, 2021.

[12] N. Pavlicek and L. Gross, "Generation, manipulation and characterization of molecules by atomic force microscopy," Nature Reviews Chemistry, vol. 1, 2017.
[13] C. Shi, D. K. Luu, Q. Yang, J. Liu, J. Chen, C. Ru, S. Xie, J. Luo, J. Ge, and Y. Sun, "Recent advances in nanorobotic manipulation inside scanning electron microscopes," Microsystems \& Nanoengineering, vol. 2, no. 1 , p. 16024 , dec 2016 .

[14] S. Fatikow, M. Bartenwerfer, and O. C. Haenssler, "Towards Robotbased Manipulation, Characterization and Automation at the Nanoscale," in 2019 International Conference on Industrial Engineering, Applications and Manufacturing (ICIEAM), no. Figure 1. IEEE, mar 2019, pp. $1-5$.

[15] N. A. Weir, D. P. Sierra, and J. F. Jones, "A Review of Research in the Field of Nanorobotics ," Sandia Report Sand2005-6808, no. October, pp. 1-50, 2005.

[16] S. Yao, H. Li, S. Pang, B. Zhu, X. Zhang, and S. Fatikow, "A Review of Computer Microvision-Based Precision Motion Measurement: Principles, Characteristics, and Applications," IEEE Transactions on Instrumentation and Measurement, vol. 70, pp. 1-28, 2021.

[17] H. McClintock, F. Z. Temel, N. Doshi, J. S. Koh, and R. J. Wood, "The milliDelta: A high-bandwidth, high-precision, millimeter-scale Delta robot," Science Robotics, vol. 3, no. 14, 2018.

[18] S. Miyashita, S. Guitron, S. Li, and D. Rus, "Robotic metamorphosis by origami exoskeletons," Science Robotics, vol. 2, no. 10, pp. 1-7, 2017.

[19] T. G. Leong, C. L. Randall, B. R. Benson, N. Bassik, G. M. Stern, and D. H. Gracias, "Tetherless thermobiochemically actuated microgrippers," Proceedings of the National Academy of Sciences, vol. 106, no. 3, pp. 703-708, jan 2009.

[20] Y. Zhu, M. Birla, K. R. Oldham, and E. T. Filipov, "Elastically and Plastically Foldable Electrothermal Micro-Origami for Controllable and Rapid Shape Morphing," Advanced Functional Materials, vol. 2003741, pp. $1-10,2020$.

[21] J.-Y. Rauch, O. Lehmann, P. Rougeot, J. Abadie, J. Agnus, and M. A. Suarez, "Smallest microhouse in the world, assembled on the facet of an optical fiber by origami and welded in the $\mu$ Robotex nanofactory," Journal of Vacuum Science \& Technology A, vol. 36, no. 4, p. 041601, jul 2018.

[22] Y. Mao, Y. Pan, W. Zhang, R. Zhu, J. Xu, and W. Wu, "Multi-DirectionTunable Three-Dimensional Meta-Atoms for Reversible Switching between Midwave and Long-Wave Infrared Regimes," Nano Letters, vol. 16, no. 11, pp. 7025-7029, nov 2016.

[23] Y. Mao, Y. Zheng, C. Li, L. Guo, Y. Pan, R. Zhu, J. Xu, W. Zhang, and $\mathrm{W}$. Wu, "Programmable bidirectional folding of metallic thin films for 3D chiral optical antennas," Advanced Materials, vol. 29, no. 19, 2017.

[24] K. Chalapat, N. Chekurov, H. Jiang, J. Li, B. Parviz, and G. S. Paraoanu, "Self-Organized Origami Structures via Ion-Induced Plastic Strain," Advanced Materials, vol. 25, no. 1, pp. 91-95, jan 2013.

[25] Z. Liu, H. Du, Z.-Y. Li, N. X. Fang, and J. Li, "Invited Article: Nano-kirigami metasurfaces by focused-ion-beam induced close-loop transformation," APL Photonics, vol. 3, no. 10, p. 100803, oct 2018.

[26] J. Li and Z. Liu, "Focused-ion-beam-based nano-kirigami: from art to photonics," Nanophotonics, vol. 7, no. 10, pp. 1637-1650, sep 2018.

[27] P. Corke, Robotics, Vision and Control, ser. Springer Tracts in Advanced Robotics. Cham: Springer International Publishing, 2017, vol. 118.

[28] Z. Yanjiang, Z. Yongde, and J. Jin, "A Method for Solving Robot Workspace Based on Matlab," Mechanical Science and Technology for Aerospace Engineering, vol. 28, pp. 1657-1666, 2009.

[29] J. Li, F. Zhao, X. Li, and J. Li, "Analysis of robotic workspace based on Monte Carlo method and the posture matrix," Proceedings of 2016 IEEE International Conference on Control and Robotics Engineering, ICCRE 2016, pp. 2-6, 2016.

[30] L. Birglen, "Type synthesis of linkage-driven self-adaptive fingers," Journal of Mechanisms and Robotics, vol. 1, no. 2, pp. 1-9, 2009. 